\title{
Enhancement of the Optical Performance by Optimization of Optical Sheets in Direct-illumination LCD Backlight
}

\author{
Gyeungju Park, Young Gyu Kim, Jong Hoon Yi, and Jin Hyuk Kwon* \\ Department of Physics, Yeungnam University, Gyeongsan 712-749, Korea \\ Jae Hyun Park, Sung Hoon Kim, Byoung Ku Kim, and Jong Keun Shin \\ LG Display, Kumi, Gyungsangbuk-do 730-726, Korea \\ Hoi Sup Soh \\ CMPS, School of Advanced Material Engineering, Kookmin University, Seoul 136-702, Korea
}

(Received January 23, 2008 : revised February 25, 2009 : accepted February 25, 2009)

\begin{abstract}
The structure of the prism sheet and the reflective polarizer sheet in the direct - illumination liquid crystal display (LCD) backlight is optimized and fabricated. The apex angle of the prism sheet was changed from 90 degree to 96.5 and 101.5 degree and the angular spread of the diffuser films attached on the reflective polarizer sheet was changed from 7 to 1.5 degrees. The measured view angle, the normal luminance, and the contrast ratio from the optimized backlight unit showed improved optical performances.
\end{abstract}

Keywords: LCD backlight, Prism sheet, Reflective Polarizer

OCIS codes : (120.2040) Displays; (230.0230) Optical devices; (230.5480) Prisms

\section{INTRODUCTION}

A liquid crystal display (LCD) is basically composed of two parts: the liquid crystal panel and the backlight unit (BLU). The BLU of the direct-illumination LCD plays an important role in the optical performance of the LCD in terms of the luminance, the view angle, and the image quality. In addition, the cost of the BLU takes a large portion of the total cost of the LCD. So, improvement of the optical performance of the BLU is important in increasing the competitiveness of the LCD with other flat panel displays such as PDP or OLED [1-3].

A typical structure of the BLU of the direct-illumination LCD as shown in Fig. 1 is composed of light sources such as cold-cathode fluorescent lamps (CCFL) or light emitting diodes (LED) [4], the diffuser plate (DP), the diffuser sheet (DS), the prism sheet known as the brightness enhancement film (BEF), and the reflective polarizer sheet known as the dual brightness

*Corresponding author: jhkwon1@yu.ac.kr enhancement film (DBEF). Fig. 2 shows the internal structure of the reflective polarizer sheet (DBEF) including the core reflective polarizer layer and the diffuser films attached on both sides of the core reflective polarizer layer. Fig. 3 shows the measured change of the angular luminance profiles when the lights from the light source propagate upward passing through the optical sheets. The diffuser plate [5-6] protects the light sources and the liquid crystal panel by providing a mechanical support and also provides sufficient uniformity of light by removing the bright lines of CCFLs or hot spots of LEDs. The angular luminance profile of lights after passing the diffuser plate becomes almost a Lambertian distribution. The diffuser sheet that is composed of scattering beads distributed in the transparent resin on the top surface of a flexible sheet enhances the normal luminance and decreases the view angle or the angular luminance width [7-9].

The prism sheet increases the normal luminance about $30 \%$ by reducing the angular luminance width [10-12]. Usually a small LCD such as a notebook or a monitor needs two crossed prism sheets to achieve the needed 


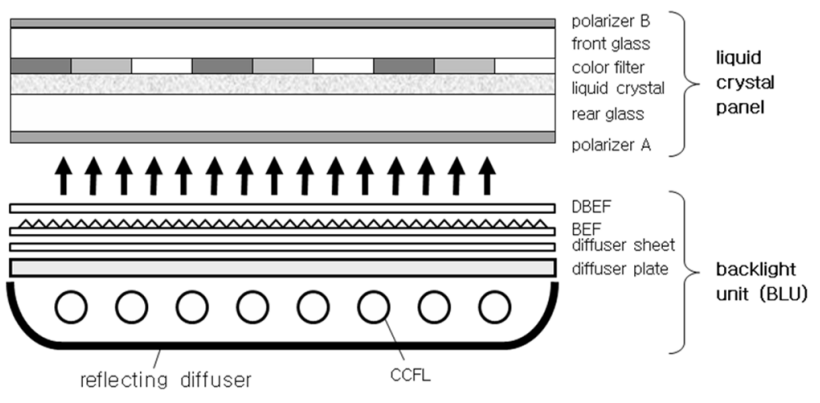

FIG. 1. The structure of LCD backlight including the diffuser plate, the diffuser sheet, the prism sheet known as the brightness enhancement film (BEF), and the reflective polarizer known as the dual brightness enhancement film (DBEF).

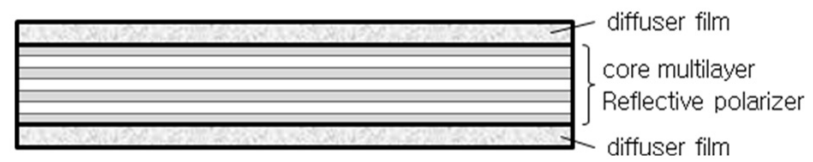

FIG. 2. The internal structure of the reflective polarizer sheet showing the core reflective polarizer with diffuser films attached on the both sides.

normal luminance by sacrificing the view angle but the direct-illumination LCDs larger than 32 inches in size, use only a single prism sheet for a wider horizontal view angle. A typical prism sheet has the apex angle of 90 degrees and there exist two strong sidelights along the vertical direction at the angles of $+/-80$ degrees from the normal direction as shown in Fig. 4. Fig. 4(a) shows the simulation results for the angular luminance profile along the horizontal direction and Fig. 4(b) shows the measured vertical angular luminance profiles. It is clearly shown in Fig.4 that there exist two strong sidelights along the vertical direction. Fig. 5 shows how the sidelights are formed in the prism sheet. There exist three groups of rays with different propagation characteristics. The first group is the rays entering the prism sheet at almost a normal direction. All of these rays are reflected after double reflections at the prism and then recycled at different incident angles. The second group of rays has oblique incidence angles. These rays are refracted twice and emerge at a normal direction with an angular width of about 60 degrees. The third group of rays has very high incidence angles and emerges as the sidelights at the angle of about 80 degrees from the normal direction. There are three issues to be improved in the prism sheet: the mechanical weakness, the narrow angular width along the vertical direction, and the unnecessary sidelights. Among these the second and third problems can be improved by modifying the structure of the prism sheet and the diffusivity of the reflective polarizer sheet.

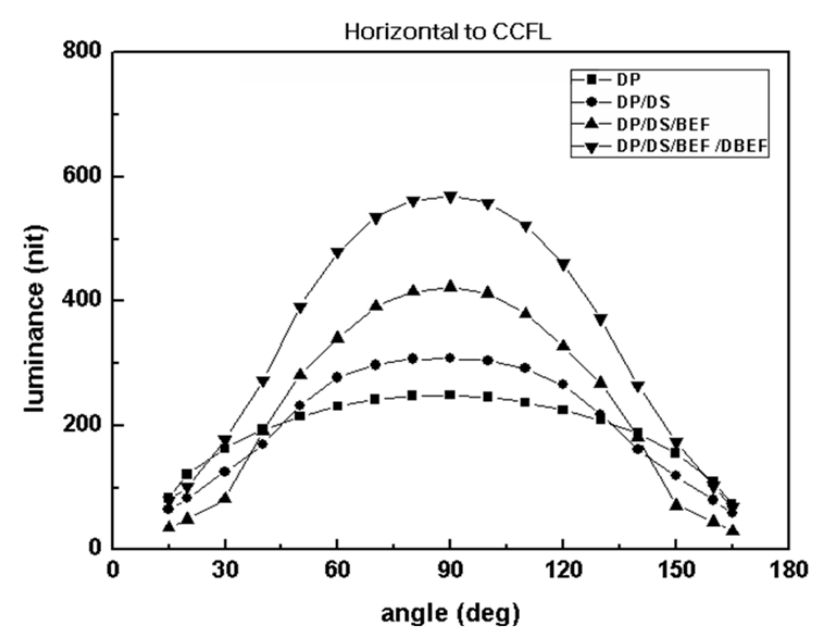

(a)

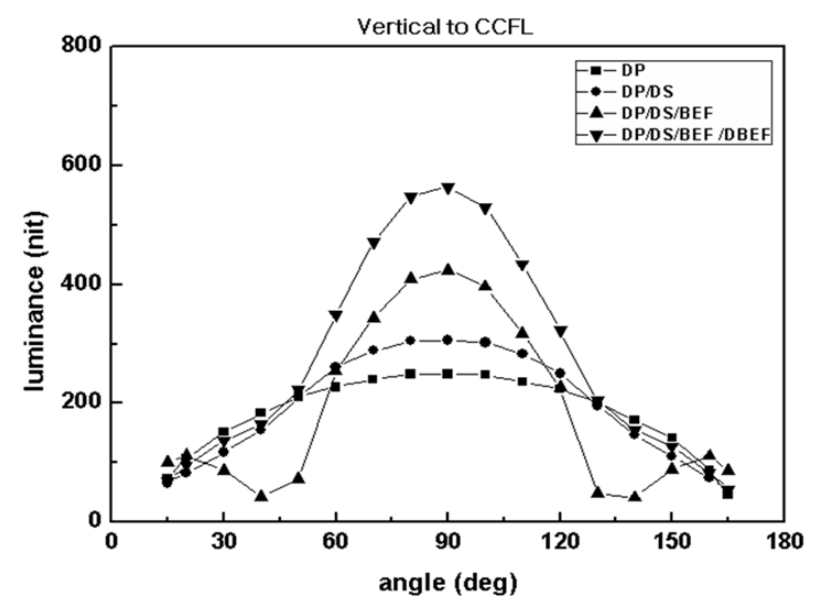

(b)

FIG. 3. The angular luminance profiles of the LCD backlight unit as the light passed through the optical sheets composed of the diffuser plate (DP), the diffuser sheet (DS), the prism sheet (BEF), and the reflective polarizer sheet (DBEF) from the light source. (a) The horizontal angular luminance profile, (b) The vertical angular luminance profile.

The reflective polarizer sheet increases the normal luminance as much as 30 40\% by polarization - recycling of the reflected light. A typical reflective polarizer, which is known as the dual brightness enhancement film (DBEF), has diffuser films attached on both sides of the core multilayer reflective polarizer layer to remove the Moire fringes between the liquid crystal panel and the prism sheet, and also to reduce the sidelights generated from the underlying prism sheet. Fig. 6 shows the reduced sidelights after passing through the reflective polarizer with diffuser films attached on the both sides.

In this paper we studied optimization of the combined structure of the prism sheet and the reflective polarizer sheet to achieve a higher normal luminance, a wider view angle, and a higher contrast ratio. The apex angle 


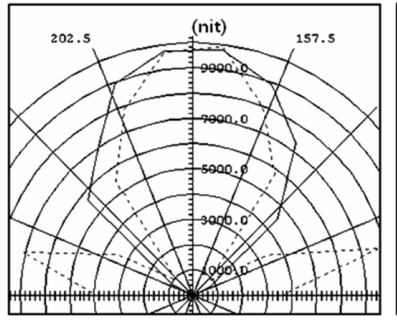

(a)

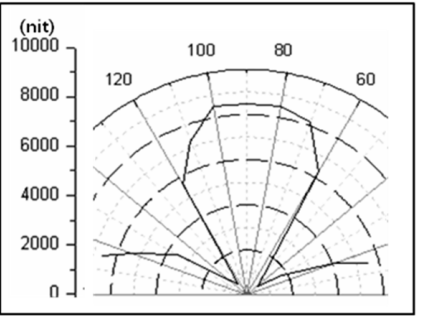

(b)
FIG. 4. The angular luminance profiles of the prism sheet. (a) the angular luminance profile obtained by simulation; solid line ; horizontal angular luminance profile, dashed line ; the vertical angular luminance profile. (b) the measured vertical angular luminance profile.

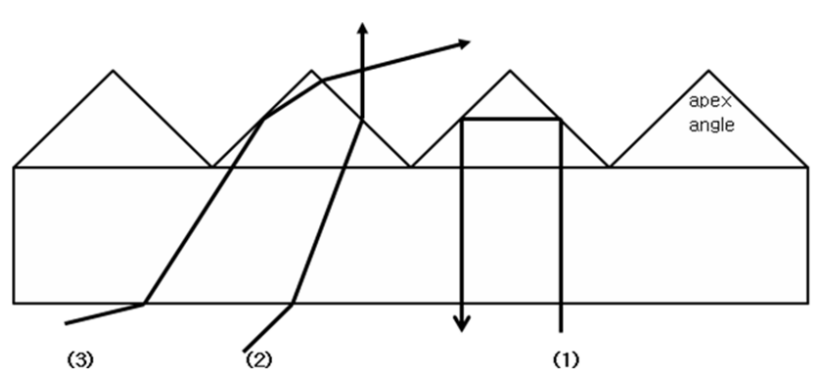

FIG. 5. The three types of light propagation in the prism sheet.

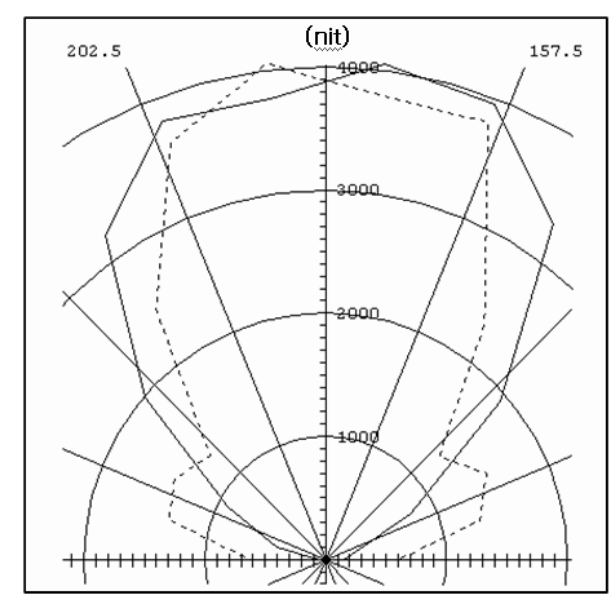

FIG. 6. The horizontal (solid line) and vertical (dashed line) angular luminance profiles.

of the prism sheet was changed from 90 degrees to 110 degrees in steps of 4 degrees and also the diffusivity of the diffuser films attached on the core multilayer reflective polarizer was changed. Prototype samples of the modified prism sheet with the optimized apex angle and the reflective polarizer with an optimized diffusivity were fabricated and their normal luminance, view angle, and contrast ratio were measured and compared with the simulation results.

\section{OPTIMIZATION}

The apex angle of the prism sheet and the diffusivity of the diffuser films attached on both sides of the core multilayer reflective polarizer were changed and the condition for the maximum normal luminance and the widest view angle were found by simulation of the backlight unit. The Light Tools program was used as the simulation tool. A 32" BLU simulation model as shown in Fig. 1 was constructed including optical components such as the diffuser plate, the diffuser sheet, the prism sheet, and the reflective polarizer sheet. The scattering characteristics of the diffuser plate and the diffuser sheet were assumed to be Lambertian and Gaussian, respectively. The base material of the diffuser plate and the diffuser sheet were assumed to be PMMA (polymethylmethacrylate) and PET (polyethylenterephthalate). The thicknesses of the diffuser plate and diffuser sheet were assumed to be $3 \mathrm{~mm}$ and $0.2 \mathrm{~mm}$, respectively. The refractive indices of the diffuser plate and the diffuser sheet are 1.49 and 1.59 , respectively. The core multilayer polarizer of the reflective polarizer was simulated by using the library polarizer component in the Light Tools program. The reflectivities of the p- and s-polarized light were assumed to be $90 \%$ and $10 \%$, respectively. Also, it was assumed that there was no absorption loss or scattering loss in the core multilayer polarizer. The 18 CCFLs were used as the light sources.

The parameters of simulation for the prism sheet and the reflective polarizer were the apex angle of the prism sheet and the diffusivity of the diffuser films attached on both sides of the core multilayer reflective polarizer. The scattering characteristics of the diffuser plate and the diffuser sheet were not changed. The normal luminance and the view angle of the direct - illumination LCD backlight were obtained from simulation for the apex angles of the prism sheet as 90, 94, 98, 102, 106 degrees.

The bidirectional scattering functions (BSDF) of the diffuser films attached on both sides of the reflective polarizer were assumed to have the Gaussian scattering function as follows:

$$
P(\theta)=P_{0} \exp \left[-\frac{1}{2} \frac{\theta^{2}}{\sigma^{2}}\right]
$$

, where $P_{0}$ is the radiance of the specular reflection, is the scattering angle in degrees from the specular direction, and $\theta$ is the diffusivity of the diffuser film also known as the Gaussian spread angle. Fig. 7 shows the measured scattering function of the diffuser film and the best fit curve was the Gaussian scattering function with $\sigma$ degree. In the simulation of the reflective polarizer the values used for the diffusivity were $11,9,7,5,3$, 1 degrees. 


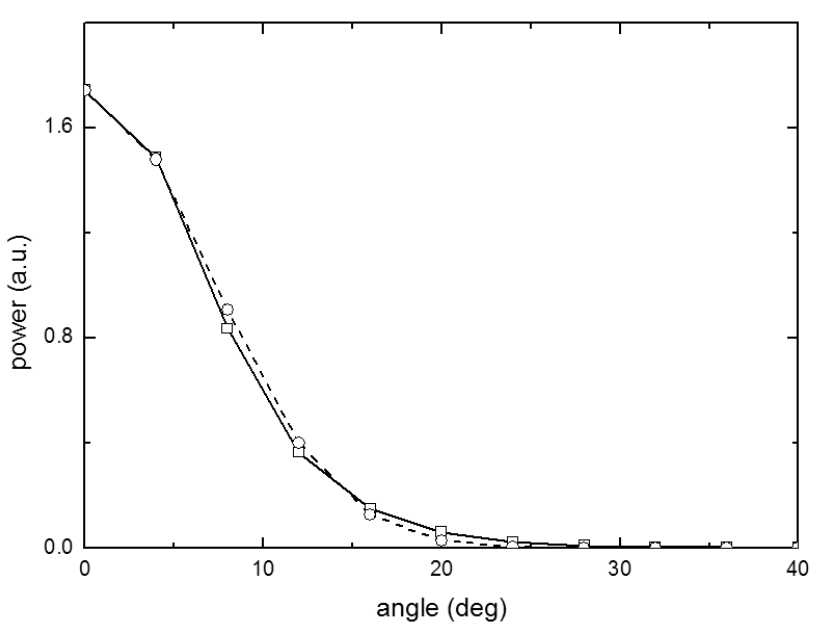

FIG. 7. The measured scattering distribution (solid line) and the best fit using the Gaussian scattering function with the angular spread $7 \mathrm{deg}$ (dashed line).

For all the combinations of the apex angles and the diffusivity values the normal luminance, the horizontal view angle, the vertical view angle and the luminance of the sidelights were obtained. However, only two values of the diffusivity $\sigma=7$ and 1.5 degrees were used because the reflective polarizer with those values were available. The reflective polarizer with $\sigma=7$ degree is the widely used model and the reflective polarizer with $\sigma=1.5$ degree is the newly developed model in which the diffuser films that were attached on both sides of the core multilayer polarizer have less scattering. The reference values of the normal luminance, the horizontal view angle, and the vertical view angle for the standard BLU were 7,000 nit $\left(\mathrm{cd} / \mathrm{m}^{2}\right) 102$ degree, and 77 degree. Fig. 8 (a) and (b) shows the change of the view angles and the normal luminance as a function of the apex angle of the prism sheet. The horizontal and vertical view angles increase monotonically as the apex angle is increased and the normal luminance shows a peak value for the apex angle of 98 degrees. The optimized values of the apex angle of the prism sheet and the diffusivity of the reflective polarizer were 98 degrees and 3 degrees with the result of the normal luminance of 7,400nit, the horizontal view angle of 110 degrees, and the vertical view angle of 78 degrees. The normal luminance went up about $5.7 \%$ higher and the horizontal view angle was $7.8 \%$ wider than for the standard BLU.

\section{FABRICATION AND MEASUREMENTS}

Two optimized prism sheets with apex angles of 96.5 and 101.5 degrees and two reflective polarizers with the diffusivity $\sigma=7$ and 1.5 degrees were fabricated and their optical performance in the LCD backlight were measured. The reason for the difference between the

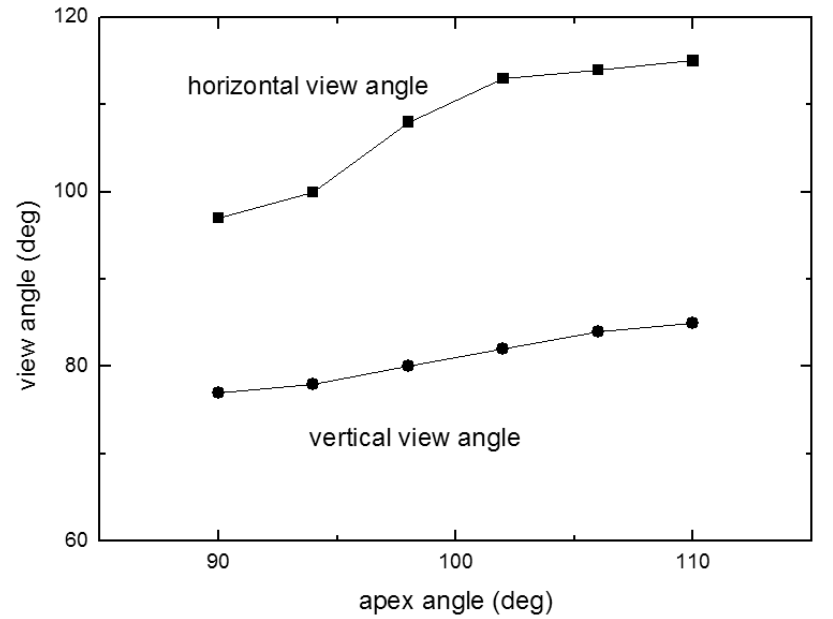

(a)

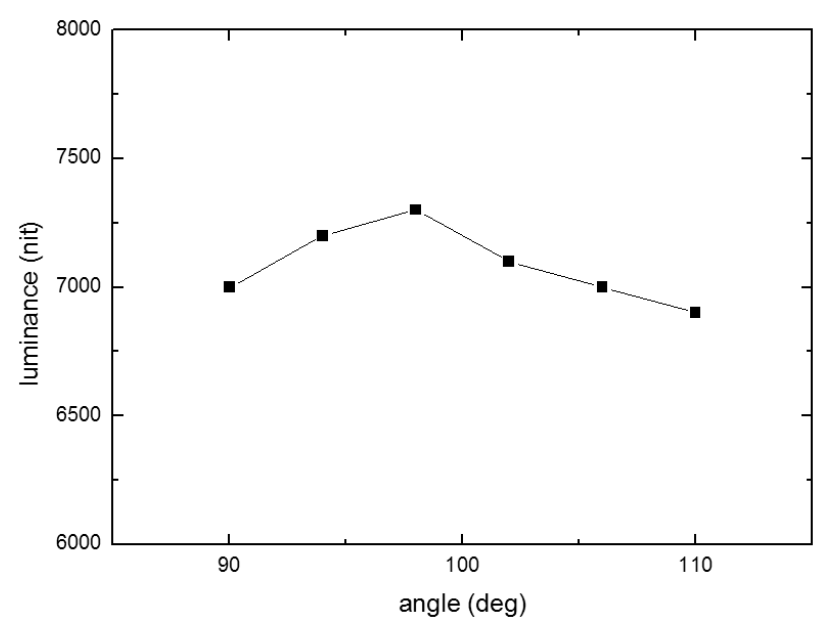

(b)

FIG. 8. (a) The horizontal and vertical view angles and (b) the normal luminance change as a function of the apex angle of the prism sheet.

simulation and that of the fabricated samples is due to the manufacturing error. Fig. 9 shows the scattering distribution of the reflective polarizes DBEF with the diffusivities $\sigma=7$ and 1.5 degrees. Table 1 shows the measured values for the normal luminance and the horizontal view angle of the backlight unit for various combinations of the standard and optimized prism sheet and the reflective polarizer. DP, DS, MBEF1, MBEF2, and MDBEF mean the diffuser plate, the diffuser sheet, the modified BEF 1 with apex angle 96.5 degrees, the modified BEF 2 with the apex angle 101.5 degree, and the modified DBEF with diffusivity 1.5 degrees, respectively. When MBEF1 and MBEF 2 were used with the current reflective polarizer with the diffusivity $\sigma=7$, the normal luminance decreased $1.2 \%$ and $2.9 \%$, respectively. Both the normal luminance and the view angle were increased when both the MBEF and the MDBEF were used together instead of the standard prism sheet 
TABLE 1. The change of normal luminance and view angle according to the combination of optical sheets including the modified brightness enhancement film (MBEF 1,2) and the modified dual brightness enhancement film (MDBEF)

\begin{tabular}{l|c|c|c|c}
\hline \hline \multicolumn{1}{c|}{ Optical Sheet Stack } & Normal Luminance & Difference & View Angle & Difference \\
\hline DP/DS/BEF/DBEF & 6938 & 0 & 106.5 & 0 \\
\hline DP/DS/MBEF1/DBEF & 6852 & $-1.2 \%$ & 111.6 & $5.1 \%$ \\
\hline DP/DS/MBEF2/DBEF & 6738 & $-2.9 \%$ & 116.7 & $9.6 \%$ \\
\hline DP/DS/MBEF1/MDBEF & 7267 & $4.7 \%$ & 113.1 & $6.2 \%$ \\
\hline DP/DS/MBEF2/MDBEF & 7118 & $2.6 \%$ & 116.2 & $9.1 \%$ \\
\hline
\end{tabular}

TABLE 2. The change of normal luminance for white and black mode and the contrast ratio according to the combinations of the optical sheets including the modified brightness enhancement film (MBEF 1,2) and the modified dual brightness enhancement film (MDBEF)

\begin{tabular}{l|c|c|c|c|c}
\hline \hline & $\begin{array}{c}\text { White } \\
\text { luminance }\end{array}$ & $\begin{array}{c}\text { luminance } \\
\text { difference }\end{array}$ & Black luminance & $\begin{array}{c}\text { Contrast Ratio } \\
\text { (CR })\end{array}$ & CR difference \\
\hline DP/DS/BEF/DBEF & $546.3 \pm 1.2$ & $0 \%$ & 0.8 & 682.9 & $0 \%$ \\
\hline DP/DS/MBEF1/DBEF & $544.9 \pm 3.3$ & $-0.3 \%$ & 0.8 & 681.1 & $0.2 \%$ \\
\hline DP/DS/MBEF2/DBEF & $547.8 \pm 0.8$ & $0.3 \%$ & 0.8 & 684.3 & $0.2 \%$ \\
\hline DP/DS/MBEF1/MDBEF & $591.8 \pm 2.5$ & $8.3 \%$ & 0.8 & 739.9 & $8.3 \%$ \\
\hline DP/DS/MBEF2/MDBEF & $579.1 \pm 1.8$ & $6.0 \%$ & 0.8 & 723.9 & $6 \%$ \\
\hline
\end{tabular}

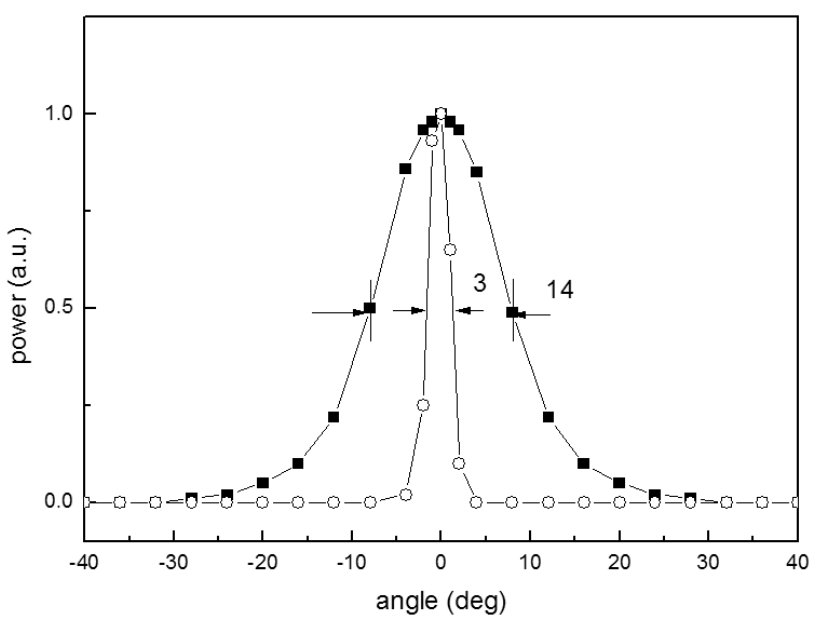

FIG. 9. The angular spread of the reflective polarizer with diffuser films of different diffusivity. The square data and circle data show the angular spreads for the diffusivity $\sigma=7$ and 1.5 , respectively.

(BEF) and the reflective polarizer (DBEF). The normal luminance and the view angle were increased $4.7 \%$ and $6.2 \%$. Comparing these values with the simulation results of $5.7 \%$ and $7.8 \%$ the measurement showed a close agreement with the simulation.

Table 2 shows the measured normal luminance and the contrast ratio measured above the liquid crystal panel for various combinations of the standard and optimized prism sheets and reflective polarizers. When both the optimized MBEF1 and the MDBEF were used the normal luminance was increased $8.3 \%$ from 546.3nit to 591.8nit and also the contrast ratio was increased $8.3 \%$ from 682.9 to 739.9 . It seems that both the normal luminance and the contrast ratio were increased due to the less scattering of the modified DBEF.

\section{CONCLUSION}

An optimized structure of the prism sheet and a modified reflective polarizer with diffuser films with less scattering were designed and fabricated. The measured normal luminance, the view angle, and the contrast ratio showed improved performance compared to the previous backlight unit. This shows that the optical performance of the LCD can be improved without any additional cost. by adopting the optimized prism sheet and the reflective polarizer with smaller diffusivity

\section{ACKNOWLEDGMENT}

This research was supported by the Yeungnam University research grants in 2008.

\section{REFERENCES}

1. M. Stewart, R. S. Howell, L. Pires, and M. K. Hatails, "Polysilicon TFT technology for active matrix OLED displays," IEEE Transactions on electron devices 48, 845-851 (2001).

2. J. C. Sturm and C. C. Wu, "Integrated organic light 
emitting diode structures using doped polymer," in Proc. IDRC Annual Meeting (1997), pp. F11-F18.

3. H. Hovagimian and J. Mehlhaff, "Recent advances in rapid thermal processing of polysilicon TFT LCD's, in Proc. IDRC Annual Meeting (1997), pp. M52.

4. I. K. Kim and K. Y. Chung, "Wide color gamut backlight from three-band white LED,” J. Opt. Soc. Korea 11, 67-70 (2007).

5. A. Tagaya, S. Ishii, K. Yokoyama, Higuchi E, and Koike $\mathrm{Y}$, "The advanced highly scattering optical transmission polymer backlight for liquid crystal,” Jpn. J. Appl. Phys. 41, 2241-2248 (2002).

6. G. H. Kim, "A PMMA composite as an optical diffuser in a liquid crystal display backlight unit (BLU)," European Polymer Journal 41, 1729-1737 (2005).

7. G. J. Park, T. S. Aum, D. H. Lee, J. H. Kwon, M. H. Lee, B. K. Jung, and H. S. Soh, "Enhancement of luminance and view angle by optimizing the structure of prism sheet and the diffusivity of reflective po- larizer film," in Proc. IDW Annual Meeting (Otsu, Japan, Dec. 2006), pp. 953-955.

8. Y. H. Ju, J. H. Park, J. H. Lee, J. Y. Lee, K. B. Nahm, J. H. Ko, and J. H. Kim, "Study on the simulation model for the optimization of optical structures of edge - lit backlight for LCD applications," J. Opt. Soc. Korea 12, 25-30 (2008).

9. G. J. Park, T. S. Aum, J. H. Kwon, J. H. Park, B. K. Kim, and J. K. Shin, "Characterization and modeling light scattering in diffuser sheets," J. Korean Phys. Soc. 54, 44-48 (2009).

10. M. Edward, M. B. O’Neill, and D. L. Wortman, US Patent 6091547 (2000).

11. A. C. Hamilton and J. Courtial, "Optical properties of a dove-prism sheet," J. opt. A: Pure and applied optics 10, 125302-125307 (2008).

12. A. Abileah and ND Vergith, "Liquid crystal display with holographic diffuser and prism sheet on viewer side," U.S. patent 5629784 (1997). 